\title{
Impiego del sistema URO-QUICK per l'esecuzione rapida di antibiogrammi direttamente su campioni di urine
}

\author{
Eugenio A. Debbia, Simona Roveta, Anna Marchese
}

Sezione di Microbiologia - DISCAT

Università degli studi di Genova - Largo Rosanna Benzi 10 -I6/32 Genova

Key words: Uro-Quick, antibiotic susceptibility test in urine

\section{SUMMARY}

During the period june-october 2003 the urine samples were examined employing routine methods for strain identification and the Kirby-Bauer technique for antibiotic susceptibility tests. This usual system was compared with the new rapid Uro-Quick method employed on samples resulting positive and mono-microbial after Gram coloration. Antibiotic (in appropriate concentration) was introduced in a vial containing $2 \mathrm{ml}$ of Mueller-Hinton broth, then $0.5 \mathrm{ml}$ of urine were added in each vial containing the antimicrobial molecules and even in a vial without drug used as control. After 3-5 hours of incubation (for Gram negative or Gram positive strains respectively) the instrument shows the results. No growth and a growth curve like the control are representative of a susceptible and resistant strain respectively). Gram negative strain were tested against ciprofloxacin, nitrofurantoin, co-clavulanate, ceftazidime, fosfomycin, imipenem, amikacin, and trimethoprim-sulfamethoxazole, while Gram positive bacteria against ciprofloxacin, nitrofurantoin, co-clavulanate, ampicillin, fosfomycin, gentamycin, oxacillin and trimethoprim-sulfamethoxazole. The Gram negative strains isolated were 1172 and the Gram positive were 261 . With the first group agreement between the two methods was always more than $90 \%$, against Gram positive pathogens there was more than $80 \%$ of agreement. In conclusion against the mayor urinary tract pathogens (E. coli, Enterococci, Klebsiella spp. and Proteus spp.) agreement between the Uro-Quick system and the KirbyBauer was more than $90 \%$. The rapid method appears useful not only for the determination of the antibiotic susceptibility of common uropathogens, but, on the basis of the present findings, it could be suggested the use of this rapid method in more severe nosocomial infections.

\section{INTRODUZIONE}

Le infezioni delle vie urinarie (IVU) rappresentano una delle patologie più diffuse a livello mondiale, indipendentemente dal tipo di popolazione comunitaria o nosocomiale. Per questo motivo l'urinocoltura è tuttora l'esame batteriologico più richiesto al laboratorio di microbiologia. La prevalenza delle IVU varia con l'età e il sesso e può essere condizionata dalla presenza di alcuni fattori predisponenti, nell'età adulta è più frequente nelle donne mentre nella popolazione anziana si presenta con uguale frequenza sia negli uomini che nelle donne. Circa il 5\% di tutte le donne va incontro ad almeno un episodio di IVU prima dei 20-24 anni e si stima che almeno il $20 \%$ della popolazione femminile presenti un episodio di cistite ogni anno $(8,14,16)$ : proprio questa patologia dal punto di vista epidemiologico costituisce 1 ' $80 \%$ di tutte le infezioni urinarie. Di fronte ad un caso isolato di cistite è in genere sufficiente instaurare una terapia antibiotica su base empirica, mirata contro i patogeni più frequentemente responsabili dell'infezione (E. coli, Enterococcus spp., Klebsiella spp., Proteus spp.) (1, 4, 5, 7). Il razionale di tale strategia terapeutica si basa non solo sulla conoscenza del limitato numero di agenti eziologici che causano la cistite ma anche sulla conoscenza delle loro percentuali di resistenza agli antibiotici comunemente usati per il trattamento di queste infezioni. Occorre sottolineare che lo sviluppo di resistenze agli antimicrobici varia in funzione delle specie batteriche considerate, dei contesti clinici in cui si sviluppano (ambiente ospedaliero oppure comunitario) e nell'ambito della distribuzione geografica $(8,14,16$, 17). É necessario pertanto avere dati sempre aggiornati sull'incidenza delle resistenze. In certe situazioni, inoltre, la rapida ed esatta conoscenza dell'insensibilità dei patogeni agli antibiotici può essere fondamentale per giungere ad una rapida guarigione del paziente, scongiurando così un uso improprio degli antibiotici che sta alla base dell'aumento delle resistenze e dei costi. In questo contesto può essere di fondamentale aiuto uno strumento come l'Uro-Quick impiegato da tempo per la valutazione del numero di batteri presenti nell'urina in un tempo che si approssima alle 3 ore $(6,10,15)$. In studi preliminari è stato inoltre 
accertato che il saggio di alcuni antibiotici può essere effettuato direttamente sui patogeni contenuti nel campione di urina $(12,13)$. Questo studio si propone di utilizzare il sistema Uro-Quick per la determinazione rapida della sensibilità agli antibiotici su un ampio numero di campioni urinari positivi e monomicrobici (più del $95 \%$ delle IVU sono dovute a una singola specie batterica) e di determinare la concordanza dei risultati con quelli ottenuti con la metodica usuale del KirbyBauer.

\section{MATERIALI E METODI}

Nel periodo giugno-ottobre 2003 sono stati esaminati in modo routinario per la presenza e la conta dei microrganismi mediante sistema UroQuick 2037 campioni di urina di provenienza sia nosocomiale che comunitaria. Sui campioni risultati positivi e monomicrobici (1433) dopo screening con colorazione di Gram è stato eseguito l'esame colturale per l'identificazione dei germi secondo le procedure tradizionali con l'impiego di prove metaboliche e biochimiche.

L'antibiogramma è stato eseguito direttamente sulle urine mediante il sistema Uro-Quick e i risultati ottenuti sono stati confrontati con quelli relativi alla metodica tradizionale della diffusione da dischetto di Kirby-Bauer (9).

Il metodo Uro-Quick per la determinazione della sensibilità agli antibiotici prevede l'utilizzo dell'urina intera quando la carica batterica risulta minore di $3 \times 10^{6} \mathrm{CFU} / \mathrm{ml}$, mentre per concentrazioni maggiori di $3 \times 10^{6} \mathrm{CFU} / \mathrm{ml}$ occorre effettuare una diluizione 1:5 del campione con soluzione fisiologica. Se il numero di microrganismi risulta incluso tra 10.000 e 100.000 il campione viene concentrato 10 volte per centrifugazione.

Quest'ultima modificazione si è resa necessaria specialmente per stafilococchi ed enterococchi. Il sistema prevede l'aggiunta di ciascun antibiotico da saggiare direttamente ai $2 \mathrm{ml}$ di terreno eugonico contenuto nelle apposite cuvette Uro-Quick: la concentrazione di farmaco da utilizzare è stata calcolata sulla base dei breakpoints suggeriti dall'NCCLS in modo che il valore fosse compreso nell'intervallo di sensibilità intermedia. Il campione da analizzare $(0.5 \mathrm{ml})$ è dispensato nelle cuvette Uro-Quick che vengono successivamente inserite nello strumento. La lettura finale viene effettuata dopo 3 (Gram-negativi) o 5 (Gram-positivi) ore. Il ceppo è considerato sensibile in assenza di sviluppo, resistente se la curva di crescita è analoga a quella del controllo senza antibiotico.

Verso i Gram-negativi sono stati saggiati ciprofloxacina (CIP), nitrofurantoina (FT), fosfomicina (FOS), amoxicillina-clavulanato (AMC), ceftazidime (CAZ), imipenem (IPM), amikacina (AN) e cotrimossazolo (SXT). Per quanto riguarda i Gram-positivi gli antibiotici saggiati sono stati: CIP, FT, AMC, ampicillina (AMP), FOS, SXT, gentamicina (GM) e oxacillina (OXA). Le polveri e i dischetti degli antibiotici utilizzati sono stati ottenuti da fonti commerciali (SIGMA e BIOMERIEUX, Milano). Le soluzioni madre sono state allestite secondo le istruzioni del fornitore. Nella tabella 1 sono riportati l'elenco degli antibiotici, i rispettivi breakpoints e le concentrazioni utilizzate nella cuvetta Uro-Quick.

\section{RISULTATI}

Sono stati analizzati 1229 campioni di urina nosocomiali e 204 comunitari risultati positivi e monomicrobici dopo lo screening preliminare. La distribuzione dei ceppi suddivisi in Gram-negativi e Gram-positivi è riportata nelle tabelle 2 e 3 rispettivamente.

La lista dei farmaci da saggiare (tabella 1) è stata estratta dal gruppo dei più comuni antibiotici impiegati per la terapia delle infezioni urinarie sostenute da ceppi sia Gram-positivi che Gramnegativi. La concentrazione di antibiotico impiegata per il saggio è stata quella che, dopo alcune prove iniziali in cui sono state saggiate diverse concentrazioni di antibiotico comprese tra i valori di breakpoint, ha fornito la migliore riproducibilità di risultati in accordo con quelli ottenuti con la metodica classica del Kirby-Bauer.

Nella tabella 4 sono riportate le percentuali di concordanza tra i dati ottenuti con il sistema UroQuick in confronto con il Kirby-Bauer sui ceppi Gram-negativi. Tra questi E. coli, che rappresenta il patogeno più frequentemente isolato, mostra sempre concordanze superiori al $90 \%$; i migliori risultati sono stati ottenuti con AN $(99.3 \%)$, IPM (99\%), FOS (98.4\%), CIP (98.4\%), FT (97.5\%) e SXT (95\%). Globalmente si riscontrano sempre percentuali di accordo superiori all' $80 \%$ relativamente a tutte le specie, con la sola eccezione di Pseudomonas spp. Nella tabella 5 i microrganismi Gram-negativi sono suddivisi in base alla provenienza in nosocomiali e comunitari e viene riportata infine la percentuale di concordanza sulla totalità dei ceppi. I dati più soddisfacenti sono stati ottenuti relativamente a IPM $(98.2 \%)$ e CIP (96.7\%), questi due farmaci hanno fatto riscontrare addirittura una concordanza del 100\% sui campioni comunitari. Anche AN (96.3\%) e FT (95.6\%) mostrano buone percentuali di concordanza, anche se le percentuali più alte sono state riscontrate sui ceppi comunitari $(98.4 \%$ e $99.4 \%$ rispettivamente). Sia sui ceppi nosocomiali che su quelli comunitari comunque sono state sempre evidenziate nell'insieme della totalità delle specie concordanze percentuali non inferiori al $90 \%$. 
Nelle Enterobacteriaceae prevalgono le discordanze del tipo Kirby-Bauer sensibile/Uro-Quick resistente, specialmente a carico dei ß-lattamici e degli aminoglicosidi. Il fenomeno opposto si verifica invece in Pseudomonas spp.; questo potrebbe essere dovuto alla difficoltà di crescita di questi microrganismi in scarsità di ossigeno, che è l'ambiente presente nella cuvetta del sistema UroQuick.

Per quanto riguarda i Gram-positivi (tabella 6) sono state riscontrate concordanze sempre superiori all' $80 \%$. Sull'enterococco, che predomina nettamente sullo stafilococco, i migliori risultati sono stati ottenuti per FOS e AMP $(97.8 \%$ e $96.7 \%$ ), un buon accordo tra i dati ottenuti con le due metodiche è stato osservato anche per FT e CIP (94.5\% e $87.9 \%$ rispettivamente). Nello stafilococco sono state evidenziate percentuali di concordanza superiori all' $85 \%$ per CIP, OXA e AMC, maggiori del $90 \%$ per SXT e superiori al $95 \%$ per FT e FOS. Complessivamente nei ceppi nosocomiali si è avuta una corrispondenza maggiore del $90 \%$ per FT $(96.4 \%)$, AMP $(96.5 \%)$, FOS (97.1\%) e SXT (93\%). Nei ceppi comunitari le metodiche hanno dato risultati sovrapponibili nel $100 \%$ dei casi per FT e FOS (tabella 7).

Nei ceppi Gram positivi si riscontra sempre una netta prevalenza di discordanze del tipo KirbyBauer resistente/ Uro-Quick sensibile per i chinoloni, molti di questi a carico di enterococchi con resistenza intermedia alla ciprofloxacina che presentavano difficoltà di interpretazione anche con la metodica classica. È stato invece osservato un maggior numero di discordanze del tipo KirbyBauer sensibile/Uro-Quick resistente a carico di FT, FOS, SXT e aminoglicosidi.

Tabella I. Breakpoints degli antibiotici impiegati in questo studio e concentrazioni utilizzate nella cuvetta Uro-Quick

\begin{tabular}{lll}
\hline ANTIBIOTICO & $\begin{array}{l}\text { BREAKPOINT CONCENTRAZ. UQ } \\
\text { MG/L }\end{array}$ & MG/L \\
\hline Amoxicilina (Gram negativi) & $32-8$ & 15 \\
\hline Clavulanato (Gram negativi) & $16-4$ & 10 \\
\hline Amoxicillina (Staphylococcus spp.) & $8-4$ & 6 \\
\hline Clavulanato (Staphylococcus spp.) & $4-2$ & 3 \\
\hline Ceftazidime & $32-8$ & 20 \\
\hline Imipenem & $16-4$ & 7 \\
\hline Nitrofurantoina & $128-32$ & 40 \\
\hline Amikacina & $32-16$ & 20 \\
\hline Ciprofloxacina & $4-1$ & 1.5 \\
\hline Fosfomicina & $256-64$ & 70 \\
\hline G6P & & 50 \\
\hline Trimethoprim & $8-2$ & 6 \\
\hline Sulfametossazolo & $152-38$ & 100 \\
\hline Gentamicina & $4-16$ & 10 \\
\hline Oxacillina (S.aureus) & $2-4$ & 3 \\
\hline Oxacillina (SCN)* & $0.25-0.5$ & 0.3 \\
\hline *Stafilococchi coagulasi negativi & \\
G6P, glucosio 6 fosfato &
\end{tabular}

Tabella 2. Distribuzione dei I/ 72 ceppi Gram-negativi

\begin{tabular}{lccc}
\hline SPECIE & N. CEPPI & N. CEPPI & N. CEPPI \\
BATTERICA & NOSOCOMIALI & COMUNITARI & TOTALE \\
\hline E. coli & 460 & 140 & 600 \\
\hline Proteus spp. & 190 & 20 & 210 \\
- P. mirabilis & 156 & 20 & 176 \\
- P. vulgaris & 34 & 0 & 34 \\
\hline Klebsiella spp. & 140 & 15 & 155 \\
- K. pneumoniae & 111 & 14 & 125 \\
- K. oxytoca & 29 & 1 & 30 \\
\hline P. aeruginosa & 100 & 5 & 105 \\
\hline M. morganii & 35 & 2 & 37 \\
\hline Enterobacter spp. & 28 & 2 & 30 \\
- E. cloacae & 24 & 2 & 26 \\
- E. aerogenes & 4 & 0 & 4 \\
\hline P. stuartii & 15 & 0 & 15 \\
\hline B. cepacea & 10 & 0 & 10 \\
\hline Citrobacter spp. & 8 & 0 & 8 \\
$\bullet$ C. freundii & 7 & 0 & 7 \\
$\bullet$ C. koseri & 1 & 0 & 1 \\
\hline S. maltophila & 1 & 0 & 1 \\
\hline S. marcescens & 1 & 0 & I \\
\hline TOTALE & $\mathbf{9 8 8}$ & $\mathbf{1 8 4}$ & $\mathbf{I I 7 2}$ \\
\hline & & & \\
\hline
\end{tabular}

Tabella 3. Distribuzione dei 26 I ceppi Gram-positivi

\begin{tabular}{lccc}
\hline SPECIE & N. CEPPI & N. CEPPI & N. CEPPI \\
BATTERICA & NOSOCOMIALI & COMUNITARI & TOTALE \\
\hline Enterococcus spp. & 170 & 12 & 82 \\
- E. faecalis & 153 & 12 & 165 \\
- E. faecium & 17 & 0 & 17 \\
\hline Staphylococcus spp. & 71 & 8 & 79 \\
- S. aureus & 33 & 2 & 35 \\
- S. epidermidis & 11 & 2 & 13 \\
- S. haemolyticus & 7 & 2 & 9 \\
- altri Stafilococchi & 20 & 2 & 22 \\
coagulasi negativi & & & \\
\hline TOTALE & $\mathbf{2 4 I}$ & $\mathbf{2 0}$ & $\mathbf{1 6 I}$ \\
\hline
\end{tabular}

Tabella 4. Concordanza percentuale tra sistema Uro-Quick e metodica classica Kirby-Bauer nei ceppi Gram-negativi ANTIBIOTICI

\begin{tabular}{lllllll}
\hline Specie batterica CIP & FT AMC CAZ FOS IPM AN SXT
\end{tabular} (n. di ceppi)

\begin{tabular}{lcccccccc}
\hline $\begin{array}{l}\text { E. coli } \\
(600)\end{array}$ & 98.4 & 97.5 & 91.7 & 93.3 & 98.6 & 99 & 99.3 & 95 \\
$\begin{array}{l}\text { Proteus spp. } \\
(210)\end{array}$ & 94.3 & - & 92.9 & 88.1 & 92.9 & 97.2 & 91.4 & 91.9 \\
\hline $\begin{array}{l}\text { Klebsiella spp. } \\
(155)\end{array}$ & 99.8 & 89.4 & 94.7 & 93.3 & 90.7 & 98 & 99.8 & 92 \\
$\begin{array}{l}\text { Pseudomonas spp. } \\
(105)\end{array}$ & -1.3 & - & 81.7 & 46.5 & 94.8 & 83.5 & 78.3 \\
\hline $\begin{array}{l}\text { M. morganii } \\
(37)\end{array}$ & 94.6 & - & 86.5 & 89.2 & 91.9 & 100 & 100 & 91.9 \\
$\begin{array}{l}\text { Enterobacter spp. } \\
(30)\end{array}$ & 96.7 & 90.0 & 93.3 & 93.3 & 100 & 100 & 96.7 & 96.7 \\
$\begin{array}{l}\text { P. stuartii } \\
(15)\end{array}$ & 86.7 & - & 86.7 & 93.3 & 100 & 100 & 100 & 86.7 \\
$\begin{array}{l}\text { Altro * } \\
(20)\end{array}$ & 100 & 90 & 100 & 100 & 100 & 100 & 100 & 100 \\
*10 B. cepacea, 7 C. freundii, I C. koseri, I S. maltophila, I S. marcescens.
\end{tabular}


Tabella 5. Concordanza percentuale tra sistema Uro-Quick e Kirby-Bauer nei ceppi Gram-negativi nosocomiali e comunitari ANTIBIOTICO (N. CEPPI ANALIZZATI)

\begin{tabular}{lllllllll}
\hline & CIP & FT & AMC & CAZ & FOS & IPM & AN & SXT \\
\hline Nosocomiali 96.I & 94.7 & 91.2 & 90.0 & 90.0 & 97.9 & 95.9 & 91.5 \\
& $(988)$ & $(638)$ & $(787)$ & $(988)$ & $(988)$ & $(988)$ & $(988)$ & $(988)$ \\
\hline Comunitari & 100 & 99.4 & 92.7 & 97.8 & 99.5 & 100 & 98.4 & 96.7 \\
& $(184)$ & $(159)$ & $(179)$ & $(184)$ & $(184)$ & $(184)$ & $(184)$ & $(184)$ \\
\hline Totali & 96.7 & 95.6 & 92.5 & 91.5 & 91.7 & 98.2 & 96.3 & 92.4 \\
& $(1172)$ & $(793)$ & $(1057)$ & $(1172)$ & $(1172)$ & $(1172)$ & $(1172)(1172)$ \\
\hline
\end{tabular}

Tabella 6. Concordanza percentuale tra sistema Uro-Quick e metodica classica Kirby-Bauer nei ceppi Gram-positivi.

Specie batterica CIP FT AMC AMP FOS GM OXA SXT (n. di ceppi)

\begin{tabular}{lllllllll}
\hline Enterococcus spp. & 87.9 & 94.5 & - & 96.7 & 97.8 & - & - & -
\end{tabular}

(I82)

\begin{tabular}{llllllll}
\hline Staphylococcus spp. 87.4 & 96.2 & 88.7 & - & 96.2 & 81.1 & 87.4 & 93.7
\end{tabular} (79)

Tabella 7. Concordanza percentuale tra sistema Uro-Quick e KirbyBauer nei ceppi Gram-positivi nosocomiali e comunitari. ANTIBIOTICO (N. CEPPI ANALIZZATI)

\begin{tabular}{lllllllll}
\hline & CIP & FT & AMC & AMP & FOS & GM & OXA & SXT \\
\hline Nosocomiali & 87.6 & 94.6 & 88.7 & 96.5 & 97.1 & 80.3 & 87.3 & 93 \\
& $(24 I)$ & $(24 I)$ & $(7 I)$ & $(170)$ & $(24 I)$ & $(7 I)$ & $(7 I)$ & $(7 I)$ \\
\hline Comunitari & 90 & 100 & 87.5 & 100 & 100 & 87.5 & 87.5 & 100 \\
& $(20)$ & $(20)$ & $(8)$ & $(12)$ & $(20)$ & $(8)$ & $(8)$ & $(8)$ \\
\hline Totali & 87.7 & 95.0 & 88.6 & 96.7 & 97.3 & 81.0 & 97.3 & 93.7 \\
& $(26 I)$ & $(26 I)$ & $(79)$ & $(182)$ & $(26 I)$ & $(79)$ & $(79)$ & $(79)$ \\
\hline
\end{tabular}

\section{CONCLUSIONI}

Nell'eziologia delle IVU prevalgono i bacilli Gram-negativi, di origine gastro-enterica, di conseguenza $E$. coli è il responsabile della maggior parte delle infezioni, soprattutto in ambiente comunitario. In ambito nosocomiale invece possono assumere maggior rilievo anche altre specie batteriche di origine ambientale (acquisizione della popolazione microbica ospedaliera da parte del paziente), pertanto può diventare più difficile predire la risposta agli antibiotici di questi germi. In tali casi diventa ancor più importante effettuare un'urinocoltura con antibiogramma in breve tempo per poter trattare l'infezione con un antibiotico a cui l'agente eziologico in causa è sensibile. Nei laboratori che utilizzano metodiche tradizionali difficilmente i risultati dei test di sensibilità sono disponibili prima di $24-48$ ore dal momento del prelievo del campione e, di conseguenza, la decisione terapeutica spesso si basa sui dati epidemiologici disponibili. Questo comporta che nella maggior parte delle situazioni cliniche il dato proveniente dall'antibiogramma incide in misura piuttosto ridotta sulla terapia del singolo paziente, ma viene utilizzato per perfezionare o correggere l'iniziale scelta terapeutica.

Impiegando il sistema Uro-Quick che consente l'esecuzione rapida dell'antibiogramma direttamente sul campione d'urina in un periodo di tempo complessivo di 6-8 ore è possibile instaurare tempestivamente la terapia più appropriata riducendo così il rischio di fallimenti terapeutici. La valutazione della sensibilità agli antibiotici mediante sistema Uro-Quick ha mostrato sia sui ceppi Gram-negativi che su quelli Gram-positivi rappresentativi dei più importanti patogeni urinari nosocomiali e comunitari $(E$. coli, Klebsiella spp., Proteus spp. ed enterococco) una concordanza mediamente superiore al $90 \%$ con i dati ottenuti utilizzando il metodo tradizionale della diffusione da dischetto di Kirby-Bauer. Questo dato appare ancor più interessante considerando che i saggi sono stati condotti direttamente sul campione di urine, senza essere a conoscenza a priori della particolare specie patogena. Nei Gram-negativi sono stati ottenuti risultati migliori sulle Enterobacteriaceae piuttosto che su Pseudomonas spp., comunque la lieve disparità di dati tra sistema Uro-Quick e il Kirby-Bauer in questo microrganismo non appare molto rilevante anche in considerazione del fatto che Pseudomonas non è tra gli uropatogeni più frequenti. In alcuni esperimenti preliminari, tuttavia, la cuvetta è stata arricchita di ossigeno consentendo una crescita più rapida di questo patogeno facendo registrare una concordanza totale tra $\mathrm{i}$ due sistemi anche in tempi inferiori alle 24 ore (Cavallini, et al. Dati non pubblicati). Per quanto riguarda i Gram-positivi una problematica è costituita dagli enterococchi con resistenza intermedia alla ciprofloxacina, il problema è infatti apparso più evidente nei ceppi di provenienza nosocomiale rispetto a quelli comunitari in cui questo tipo di resistenza appare meno diffusa. Le poche discrepanze osservate negli stafilococchi possono essere imputate in gran parte alla scarsa capacità di alcune specie di adattarsi al terreno colturale.

I sistemi automatizzati o semiautomatici possono risentire maggiormente rispetto alle metodiche tradizionali dell'influenza di alcuni fattori (inoculo, specie batterica considerata, temperatura, tempo di incubazione, concentrazione del farmaco) che influiscono sul corretto accertamento della resistenza agli antibiotici $(2,3)$. Il sistema Uro-Quick è duttile e consente pertanto la modificazione di diversi parametri. Sperimentando alcune variazioni sul tempo di incubazione e sulla concentrazione finale di farmaco nella cuvetta, in modo da avere condizioni ottimali per le singole combinazioni antibiotico-patogeno, è stato osservato, in specie batteriche veicolanti meccanismi ben caratterizzati di resistenza agli antibiotici, un incremento della riproducibilità dei dati fino a una concordanza del $100 \%$ con la metodica classica (11).

Alla luce dei risultati ottenuti sono possibili le seguenti considerazioni: il sistema Uro-Quick 
consente in tempi brevi di ottenere una risposta sulla positività o negatività del campione e sulla sensibilità agli antibiotici dei ceppi eventualmente presenti, con notevoli benefici finalizzati a un'attivazione rapida di una terapia mirata. Questi dati suggeriscono una possibilità di utilizzo di questo strumento non solo nell'ambito delle infezioni urinarie, ma anche in casi più gravi in cui diventa ancor più importante valutare rapidamente la sensibilità agli antibiotici.

Ringraziamenti: gli autori desiderano ringraziare Fabrizio Cavallini e Claudia Zarra per l'aiuto dato durante alcune fasi di questo studio.

\section{BIBLIOGRAFIA}

1. Barret SP, Savage MA, Rebec MP, Guyot A, Andrews N, Shrimpton SB. Antibiotic sensitivity of bacteria associated with community-acquired urinary tract infection in Britain. J Antimicrob Chemother 1999; 44(3) : 359-65.

2. Felmingham D, Brown DFJ. Instrumentation in antimicrobial susceptibility testing. J Antimicrob Chemother 2001; 48 (Suppl S1): 81-5.

3. Ferraro MJ, Jorgensen JH. Susceptibility testing instrumentation and computerized expert systems for data analysis and interpretation. Murray PR, et al.: Manual of Clinical Microbiology, Seventh Edition, ASM Press 1999; 1593-600.

4. Fluit C, Jones ME, Schmitz FJ, Acar J, Gupta R. Antimicrobial resistance among urinary tract infection (UTI) isolates in Europe: results from the SENTRY antimicrobial surveillance proGram 1997. Antoine van Leeuwenhoek 2000; 77: 147-52.

5. Gales AC, Jones RN, Gordon KA, et al. Actvity and spectrum of 22 antimicrobial agents tested against urinary tract infection pathogens in hospitalized patients in Latin America: report from the second year of the SENTRY antimicrobial surveillance proGram. J Antimicrob Chemother 2000; 45(3): 295-303.

6. Iverson D, Fayette R, Johnson C, Shigei J, Peterson E, Pezzlo M. Detection of bacteriuria by a rapid, three hours automated screening method, ASM Chicago, USA, 1999: Abstr C-39.

7. Jones RN, Kugler KC, Pfaller MA, Winokur PL. Characteristics of pathogens causing urinary tract infections in hosptals in North America: results from the SENTRY antimicrobial surveillance proGram. Diagnostic Microbiol and Infect Dis 1999; 35(1): 55-63.

8. Kunin CM. Urinary tract infections, detection, prevention and management. $5^{\text {th }}$ ed. Baltimore, Williams \& Wilkins, 1997.

9. National Committee for Clinical Laboratory Standards. Performance standards for antimicrobial susceptibility testing. Twelfth informational supplement 2002; M100-S12; Vol 22. NCCLS, Wayne, PA.

10. Pezzlo M. Detection of bacteriuria by a rapid, three hours automated screening method. ASM, Chicago, USA 1999, Abstr C-39.

11. Roveta S, Marchese A, Debbia EA. Impiego del sistema Uro-Quick per l'identificazione di resistenze ben caratterizzate agli antibiotici veicolate da diverse specie batteriche. Microb Med 2003; 18: 49-55.

12. Roveta S, Debbia EA, Marchese A. Valutazione della sensibilità agli antibiotici di uropatogeni mediante sistema Uro-Quick direttamente sul campione di urine e confronto con il metodo Kirby-Bauer. GIMMOC 2003; 7: 67-74.

13. Roveta S, Balistreri MR, Marchese A, Debbia EA. Sistema rapido Uro-Quick per l'esecuzione di antibioGrammi: valutazione comparativa con il sistema Kirby-Bauer. $30^{\circ}$ Congr Naz Soc It Microbiol, Catania, 2002, Abstr 36.

14. Sobel JD, Kaye D. Urinary tract infections. In: Mandell GL, Douglas RG, Bennett JE. Principles and Practice of Infectious Diseases. Churchill Livingstone, 2000; 773-805.

15. Soro O, Schito GC, Raggi M. Performance of a new automated method for the detection of bacteriuria. $7^{\text {th }}$ ECCMID, Vienna, Austria, 1995, Abstr 1598.

16. Stamm WE, Norrby SR. Urinary tract infections: disease panorama and challenges. J Infect Dis 2001; 183: S1-S4.

17. Williams JD. Criteria for diagnosis of urinary tract infection and evaluation of therapy. Infection 1992; 4 (Suppl 4): S257-260.

\section{Eugenio A. Debbia}

Università degli Studi di Genova

DISCAT - Sez. Microbiologia -

Largo Rosanna Benzi 10; 16132 Genova

$\mathrm{Tel}++$ 010-3537655; Fax ++ 010504837

E-mail: eugenio.debbia@unige.it 